\title{
Roboter am Bett und Ärzte am Computer?
}

\begin{abstract}
Hans-Florian Zeilhofer ${ }^{a}$, Sabina Heuss ${ }^{b}$
a Prof. Dr. Dr. Dr. h.c. Hans-Florian Zeilhofer, Mund-, Kiefer- und Gesichtschirurgie des Universitätsspitals Basel, Leiter Hightech-Forschungszentrum des Department Biomedical Engineering und Delegierter für Innovation der Universität Basel; Co-Founder des Schweizer Startups Advanced Osteotomy Tools (AOT), welche den Operationsroboter Carlo (Cold Ablation Robot-guided Laser Osteotome) auf den Markt brachte; b Dr. phil. Sabina Heuss forscht an der Fachhochschule Nordwestschweiz im Institute for Competitiveness and Communication zu den Themen Kommunikation im Gesundheitswesen, Robotik und Künstliche Intelligenz
\end{abstract}

Wir sind meilenweit entfernt vom autonomen Spital, in dem Roboter selbständig Diagnose stellen und Behandlungen durchführen. Aber es ist eine Frage der Zeit, wann Roboter und Künstliche Intelligenz tiefer und nachhaltiger in das Gesundheitswesen eindringen werden. Gerade deshalb erstaunt, wie zukunfts- und technologiegläubig wir zurzeit die Entwicklung verfolgen, ohne steuernd einzugreifen.

Roboter und Künstliche Intelligenz werden unser Gesundheitswesen grundlegend verändern. In manchen Bereichen schneller als wir die vollständigen Konsequenzen abschätzen und somit Chancen und Risiken evaluieren können. Selbstverständlich sind heute bereits ausgefeilte und vielseitig einsetzbare Heberoboter im Einsatz, die Patienten rasch wieder bei der Mobilisierung und Pflegefachkräfte beim Umlagern helfen. In der Spitallogistik dirigieren Roboter Wäsche, Medikamente, Essen und medizinisches Verbrauchsmaterial. In der Altenbetreuung sind Roboter in der Form von Plüschtieren Zeitvertrieb, aber auch Animation zu Interaktion und spielerischem Lernen. Bei neueren Anwendungen kommen nicht mehr nur automatisierte Systeme zum Einsatz, die einem vorgegebenen Handlungsablauf folgen (Input-Output-Schema), son-

\section{Augmented und Virtual Reality haben Einzug} gehalten, vor allem, aber nicht nur im Operationssaal.

dern selbstlernende Systeme, die sich das Wissen für den besten Handlungsablauf selbständig aneignen, also induktiv lernen. Der Handlungsausgang ist damit nicht vorhersehbar und der Lösungsansatz nicht immer nachvollziehbar. Während die Aufgaben des DaVinci-Roboters noch vollständig und jederzeit vom Operateur selbst übernommen werden können (zum Beispiel im Falle einer Fehlfunktion), können vollständig automatisierte Tätigkeiten nicht mehr von Chirurgen durchgeführt werden. Bei Systemausfall wäre ein Wechsel des Operationsverfahrens nötig.
Heute betreiben selbstlernende Systeme schnellere und fehlerfreiere Mustererkennung in der Verarbeitung grosser Datenmengen und sind dabei dem menschlichen Auge weit überlegen. Algorithmen sind bei der Erkennung eines Gehirntumors auf einem MRI präziser und schneller als Ärztinnen. Bereits ist ein System von IBM in der klinischen Testung. Es untersucht, ob der Computer im Stande ist, radiologische Untersuchungen autonom zu befunden (Ahmed, Toor, O'Neil et Friedland, 2017). Augmented und Virtual Reality haben Einzug gehalten, vor allem, aber nicht nur im Operationssaal, wo zusätzliche Daten zum Patienten in Echtzeit für den Operateur ersichtlich sind und ihn intraoperativ unterstützen. In der Spracherkennung, in der Bilderkennung und der Genomik wurden in den letzten fünf Jahren enorme Fortschritte erzielt, und weitere Umwälzungen stehen an (LeCun, Bengio et Hinton, 2015). Denn es ist ebendiese Verbindung aus Mustererkennung bei grossen Datenmengen, medizinischer Wissensaneignung durch selbstlernende Systeme und Künstlicher Intelligenz, die den Quantensprung bewirken wird.

Dabei ist es oft ein fliessender Übergang von der Entscheidungsunterstützung zur Automatisierung. Bereits 2015 wurde in einer Pilotstudie der Ansatz von Künstlicher Intelligenz benutzt, um Patienten mit einem hohen Risiko eines septischen Schocks zu identifizieren (Henry, Hager, Pronovost et Saria, 2015). Forscher der Universität Bari haben eine Software entwickelt, die mit Hilfe Künstlicher Intelligenz Alzheimer anhand von MRI-Bildern mit hoher Wahrscheinlichkeit erkennt, Jahre bevor Symptome auftre- 
ten (Amoroso, et al., 2017). Vergleiche zwischen Eingriffen durch roboterassistierte Chirurgen, durch supervidierte autonome Systeme und durch manuelle laparoskopische Weichteilchirurgie wurden in einer Studie ex vivo durchgeführt und zeigen die Überlegenheit des supervidierten autonomen Systems in diesem Setting (Shademan, et al., 2016).

Es sind unter anderem folgende Entwicklungen in der Medizin, die von der Integration Künstlicher Intelligenz profitieren könnten: der Datenzuwachs aus klinischen und nicht-klinischen, invasiven und nicht-invasiven Überwachungssystemen, die Spezialisierung bzw. Fragmentierung der Medizin sowie die personalisierte Medizin.

\section{Komplexitätsreduktion durch Roboter und selbstlernende Systeme}

Heute verlassen sich Ärztinnen und Ärzte auf Computer und Maschinen, die ihnen Daten zur Behandlung ihrer Patienten liefern. Patientinnen und Patienten auf einer Intensivstation sind an bis zu 40 Geräte angeschlossen. Im Operationssaal zeigt die Entwicklung in die gleiche Richtung. Virtual Reality und Augmented Reality werden zu unverzichtbaren Instrumenten für den Chirurgen. Mit jedem neu entwickelten Gerät nimmt die Datenmenge exponentiell zu. Gemäss einer Studie des amerikanischen Marketinganalysten IDC wird sich die globale Datenmenge in den nächsten zehn Jahren verzehnfachen von 16,1 Zettabyte im Jahre 2016 auf 163 Zettabyte im Jahre 2025 (Reinsel, Gantz et

\section{Gemäss einer Studie wird sich die globale Datenmenge in den nächsten zehn Jahren verzehnfachen.}

Rydning, 2017). Gleichzeitig gilt es, hochkomplexe Therapieschemata umzusetzen. In diesem hochkomplexen Umfeld jederzeit die Patientensicherheit als erste Priorität zu setzen und gleichzeitig Patienten mit der bestmöglichen zur Verfügung stehenden Medizin zu versorgen, wird immer herausfordernder werden. Denn es wird die menschlichen Fähigkeiten übersteigen, alle zur Verfügung stehenden Daten lückenlos, zeitnah und fehlerfrei auszuwerten und einzusetzen, ohne in der Komplexitätsreduktion von Computern unterstützt zu werden.

Auch die Spezialisierung in der Medizin und die Ausweitung des medizinischen Angebots werden von der Künstlichen Intelligenz profitieren. Verbunden mit einer Zunahme polymorbider Patienten, auch aufgrund des Fortschreitens des demographischen Wandels, führt die Spezialisierung zu einer Fragmentierung der Medizin. Die FMH bietet zurzeit 46 verschiedene Facharzttitel mit 37 Schwerpunkten für die Weiterbildung an. Ärztinnen und Ärzte sind heute zu einem grossen Teil Experten einer Subspezialität. Der Versuch der Reduzierung dieser Komplexität führt heute vielerorts über die Hausärztin. Aber von ihren mehr als 80 -jährigen Patienten haben über $65 \%$ drei oder mehr Erkrankungen, die teilweise von externen Fachärzten behandelt werden. Im Spital werden Patienten von mehreren Fachärzten aus verschiedenen Kliniken behandelt. Das Risiko besteht, dass wichtige Erkenntnisse und Informationen über den Patienten verloren gehen.

Die hohe Komplexität ist für Patienten ein enormes Sicherheitsrisiko. Entwicklungen der Patient Safety Culture zielen deshalb auch in Richtung Komplexitätsreduzierung, neben der Beschleunigung von Arbeitsabläufen und einer Steigerung der Präzision. Die systematische Untersuchung über den Einsatz von Medizinischen Entscheidungsunterstützungssystemen (CDSS, Clinical Decision Support System) in Verbindung mit EHRS (Electronic Health Record Systems) zeigt, dass der Einsatz dieser Systeme die Gefahr von Medikationsfehlern verringert, indem es Patientenbehandlungen verbessert (Jia, Zhang, Chen, Zhao et Zhang, 2016). Schlussfolgernd wird formuliert, dass E-Health-Technologien die Qualität und Sicherheit in der medizinischen Patientenbehandlung verbessern (Keasberry, Scott, Sullivan, Staib et Ashby, 2018).

Angesichts der raschen Weiterentwicklung von Medizin und Medizintechnik kann die Beherrschung der Komplexität nur über automatische Systeme erfolgen. Durch die Spezialisierungen und Subspezialisierungen gewinnt der ganzheitliche medizinische Ansatz an Bedeutung. Schlussendlich geht es um Patientensicherheit, aber auch um wirtschaftliche Aspekte. Denn insbesondere die hochspezialisierte Medizin soll in Zukunft für alle bezahlbar bleiben.

Fortschritte sind auch in der Verbindung aus Künstlicher Intelligenz und personalisierter Medizin zu Erwarten. Die personalisierte Medizin soll in Zukunft dem richtigen Patienten die richtige Medizin zur richtigen Zeit mit der richtigen Dosis verabreichen. $\mathrm{Ob}$ dieses Versprechen gehalten werden kann, steht noch offen. Die Herausforderung in der Onkologie bleibt vorerst unter anderem die bessere Übereinstimmung von Patienten mit demselben pathologischen Krankheitsbild mit den existierenden Therapiemöglichkeiten. Mit einfacheren DNA-Analysetechniken steigt die Möglichkeit, die genetischen Voraussetzungen mit den 
vorhandenen Medikamenten abzustimmen. Selbstlernende Systeme erkennen aus den Datenmengen Strukturen und Gesetzmässigkeiten, generieren aus diesen Erfahrungen wiederum neue Erkenntnisse und verbessern sich selbständig weiter. Sie werden auch in verschieden Studien benutzt, um Brustkrebspatientinnen besser und genauer zu kategorisieren. Weitere Anwendungen der Systeme finden sich in der zielgerichteten Arzneimittelfreisetzung. Dadurch verspricht man sich eine individuellere Patientenbehandlung und eine Reduzierung von Nebenwirkungen. Weitere Erkenntnisse und Fortschritte in Verbindung mit Künstlicher Intelligenz werden im Bereich des Clinical Decision Making, des Drug Repurposing/Repositioning und der Arzneimittelentwicklung erwartet. Ein Artikel von Ching und weiteren 35 amerikanischen Kollegen (2018) gibt einen umfassenden Überblick über den Status von selbstlernenden Systemen in Medizin und Biologie und zeigt Grenzen und Möglichkeiten, auch im Bereich der personalisierten Medizin auf.

\section{Klinisches Personal wird auch in Zukunft nicht durch Roboter ersetzt}

Ärztinnen und Ärzte werden nie reines Wartungspersonal für Roboter, und ersetzen lässt sich klinisches Personal auch zukünftig nicht. Diagnosestellung und Patientenbehandlung sind kein linearer Prozess, sondern setzen neben vielem anderen auch Problemlösungsstrategien, Out-of-the-box-Denken, Empathie und Kreativität voraus. Skills, die kein Computersystem jemals hervorbringen wird. Die Entscheidung, welche Behandlungsmethode die beste für den Patienten darstellt, kann nur unter Einbezug des medizinischen, ethischen, sozialen Umfelds und der vorhandenen $\mathrm{Da}-$ ten sowie im Gespräch mit dem Patienten entschieden werden. Auch wenn Künstliche Intelligenz in Zukunft mehr Daten produzieren, auswerten und bewerten wird, bleibt doch die finale Interpretation der Daten klinisch geschultem Personal überlassen.

Dennoch werfen die neusten Entwicklungen Fragen nach Regulierungen auf. Es liegt an den Expertinnen und Experten des Gesundheitssystems, die Fragen systematisch, unter medizinischen, ethischen, rechtlichen und gesellschaftlichen Gesichtspunkten zu sammeln und die Entwicklung interdisziplinär und verantwortungsbewusst zu begleiten. Pflegefachpersonen und Ärzte müssen im Umgang mit autonomen und selbstlernenden Systemen geschult sein, um ihre Prozesse soweit möglich verstehen und regulierend eingreifen zu können. Verantwortlichkeiten, zum Beispiel im
Falle eines Systemversagens oder Softwarefehlers, müssen im Vorfeld geklärt sein. Die Kommunikation zwischen Patienten und Robotern bzw. zwischen Ärztinnen und Robotern will gelernt werden. Die Datenströme machen auch vor kantonalen und landesweiten Grenzen nicht Halt, und eine europa- und weltweite Regelung macht Sinn. Sicherheitsstandards und Datenschutzüberlegungen gehören ebenso in diese Diskussion wie die Diskriminierung durch ein Forschungsdatenübermass von Patienten europäischer Abstammung, die adressiert und korrigiert werden muss. Das Zulassen von lernenden Systemen im Gesundheitswesen sollte nur unter strengsten Regeln erfolgen.

\section{Pflegefachpersonen und Ärzte müssen im} Umgang mit autonomen und selbstlernenden Systemen geschult sein.

Ziel in der Arbeit mit autonomen Systemen und Robotern muss sein, der Ärzteschaft und Pflegefachkräften die Fülle an Informationen über ihre Patienten so aufzubereiten, dass sie daraus die richtigen Schlüsse ziehen können. Sie sollen wieder mehr Zeit am Bett und nicht vor dem Computer verbringen, um die Arbeiten am und für den Patienten auszuführen, die ihnen kein System abnehmen kann. Dann kann es gelingen, die Menschlichkeit in der Medizin mit den sich anbietenden Chancen von Künstlicher Intelligenz zu stärken.

Ein Lösungsansatz besteht im Austausch der verschiedensten Forschungsgruppen, die sich mit Künstlicher Intelligenz beschäftigen. Erst langsam interessieren sich, neben den Ingenieursdisziplinen und der Medizin, weitere Disziplinen für die Roboterthematik im Gesundheitswesen, beispielsweise die Pflegewissenschaften, Rechtswissenschaften, Kommunikation und die Ethik. Die Interdisziplinarität in der Erforschung und Implementation von autonomen Systemen im Gesundheitswesen kann jedoch nur durch ein Kompetenzzentrum strukturiert werden, welches die Isolierung einzelner Fachgebiete aufbricht. Der Wunsch nach wissenschaftlicher Diskussion der oben aufgeworfenen Fragestellungen bedarf einer personellen und strukturellen Infrastruktur. Inwieweit das Gesundheitssystem in Zukunft von selbstlernenden Systemen, von Künstlicher Intelligenz und von Robotern profitieren wird, hängt von den Kompetenzen der Fachkräfte, dem Vertrauen von Ärzten, Pflegefachkräften und Patienten und von den regulatorischen, rechtlichen, ethischen und wirtschaftlichen Leitlinien ab, die wir als Gesellschaft vorgeben. 


\section{Begriffsdefinition}

Künstliche Intelligenz / Artifical Intelligence (KI/AI)

Oberbegriff für die maschinelle Verarbeitung von Daten mit Hilfe von Algorithmen. Das Ziel ist das selbständige Lernen und die Selbstverbesserung von Maschinen und damit das Abbilden von menschlicher Intelligenz. Künstliche Intelligenz kann ein Roboter, ein Chatbot, eine Sprachsteuerungsfunktion oder ein Computer sein. Zurzeit existiert erst schwache künstliche Intelligenz. Ein Teilgebiet der künstlichen Intelligenz ist Machine Learning. Mit Hilfe von künstlicher Intelligenz entstehen selbstlernende und autonome Systeme.

\section{Autonome und selbstlernende Systeme}

"Autonome Systeme sind selbstregulierende und selbstentscheidende aktive Einheiten mit dem Ziel, eigenständig und ohne menschliches Eingreifen zu arbeiten. Sie weisen Merkmale intelligenten Verhaltens auf» (Deutscher Ethikrat, 2017).

Machine Learning (ML)

Teilgebiet der KI. Mathematische Anwendung, bei der die Maschine selbständig Wissen basierend auf Erfahrungen aufbaut. Deep Learning (DL)

Teilgebiet des Machine Learning. Bei komplexer Problemstellung kommt das Deep Learning zum Einsatz. Dabei verhält sich der Algorithmus wie ein neuronales Netzwerk und wird deshalb im deutschen Sprachgebrauch manchmal künstliche neuronale Netzwerke genannt. Dadurch erkennt es auch tiefliegende Muster und verbessert sich selbständig (iteratives Lernen). Dies wird aktuell vor allem in der Sprach- und Bilderkennung eingesetzt. Deep Learning kann überwacht (Supervised Learning) oder unüberwacht (unsupervised Learning) geschehen. Bei Ersterem kontrolliert ein Autor das System und greift bei Bedarf korrigierend ein.

\section{Literatur}

- Ahmed MN, Toor AS, O'Neil K, Friedland D. Cognitive computing and the future of health care cognitive computing and the future of healthcare: the cognitive power of IBM Watson has the potential to transform global personalized medicine. IEEE pulse. 2017;8(3): 4-9.

- Amoroso N, La Rocca M, Bruno S, Maggipinto T, Monaco A, Bellotti R, Tangaro S. Brain structural connectivity atrophy in Alzheimer's disease. 2017. arXiv:1709.02369.

- Ching T, Himmelstein DS, Beaulieu-Jones BK, Kalinin AA, Do BT, Way GP, et al. Opportunities and obstacles for deep learning in biology and medicine. 2018. bioRxiv, 142760.

- Deutscher Ethikrat. Autonome Systeme. Wie intelligente Maschinen uns verändern. Paper presented at the Jahrestagung. Berlin 2017.

- Henry KE, Hager DN, Pronovost PJ, Saria S. A targeted real-time early warning score (TREWScore) for septic shock. Science translational medicine. 2015;7(299):299ra122-299ra122.

- Jia P, Zhang L, Chen J, Zhao P, Zhang M. The effects of clinical decision support systems on medication safety: an overview. PloS one. 2016;11(12):e0167683.

- Keasberry J, Scott IA, Sullivan C, Staib A, Ashby R. Going digital: a narrative overview of the clinical and organisational impacts of eHealth technologies in hospital practice. Australian Health Review. 2018;41(6):646-64.

- LeCun Y, Bengio Y, Hinton G. Deep learning. nature. 2015;521(7553): 436.

- Reinsel D, Gantz J, Rydning J (2017). Data Age 2025: The Evolution of Data to Life-Critical. Don't Focus on Big Data.

- Shademan A, Decker RS, Opfermann JD, Leonard S, Krieger A, Kim PC. Supervised autonomous robotic soft tissue surgery. Science translational medicine. 2016;8(337):337ra364-337ra364. Institute for Competitiveness and Communication Riggenbachstrasse 16 CH-4600 Olten sabina.heuss[at]fhnw.ch 